\title{
The Puzzle of V407 Cygni
}

\author{
R. Hudec
}

\begin{abstract}
We discuss the nature and recent observations (optical and gamma-rays) of symbiotics binary/Mira variable V407 Cygni. In addition we discuss another object of similar category, namely the variable star FY Aql with possible association with an eruptive gamma-ray source.
\end{abstract}

Keywords: variable stars, Mira variables, symbiotic stars, gamma-rays, gamma-ray transients.

\section{Introduction}

V407 Cyg is a symbiotic binary harboring a Mira variable, of 745 day pulsation and a possible orbital period of 43 years, at a distance of $2.5 / 3.0 \mathrm{kpc}$ and a reddening of $E(B-V)=0.5 / 0.6$ (Munari et al. 1990). Miras of such a long pulsation period are generally $\mathrm{OH} / \mathrm{IR}$ sources, with a thick dust envelope which prevents direct observation of the central star. In addition to a possible previous one in 1936 (when the object was noted for the first time by Hoffmeister 1949), V407 Cyg has recently been discovered in outburst by Nishiyama and Kabashima (2010, CBET 2199) on 2010 Mar 10.8 UT. Spectroscopic confirmation was first provided by Munari et al. (2010, CBET 2204) on 2010 Mar. 13.1 UT. They noted the emergence of the spectrum of a $\mathrm{He} / \mathrm{N}$ nova that overwhelmed the absorption spectrum of the Mira. Numerous and strong emission lines were observed, which belonged to two distinct groups. The first group, composed of sharp profiles with even narrower central absorptions, originated from the ionized slow wind of the Mira. The second group, characterized by much broader profiles for helium, nitrogen and hydrogen lines, originated from the nova fast expanding ejecta. Such a scenario was highly reminiscent of the recurrent nova RS Oph. The outburst of V407 Cyg has since then been detected also in gamma-rays (ATel 2487) and at radio wavelengths (ATel 2506, ATel 2511, ATel 2514, ATel 2536), and observed in the infrared (CBET 2210).

\section{History}

The variable star V407 Cygni was discovered by Cuno Hoffmeister at Sonneberg on astronomical astrograph plates in 1949 as a nova-like variable (Hoffmeister 1949). The object was first investigated in more detail (on astronomical sky patrol plates) by Ludwig Meinunger at the Sonneberg Observatory in 1966 (Meinunger, 1966). The star exhibited a strange outburst in 1936, followed by Mira like light variations with a period of 745 days, perhaps related to a thermonuclear event in 1936.

\section{Spectroscopy}

The spectral appearance of V407 in outburst is highly peculiar. The spectrum is completely different from those ever recorded for this object and other symbiotic Mira variables in outburst. The white dwarf companion to the Mira variable experiences an outburst similar to that of classical novae, and its ejecta move in the circumstellar environment already filled by the ionized wind of the Mira (CBET 2204).

It is worth mentioning that the $\mathrm{Li}$ abundance is certainly anomalous, about a factor of 100 higher than normal. This has been interpreted by several studies (cited in Shore et al. 2011 and Munari et al. 2011) that the red giant is likely an AGB star or, at least, massive.

\section{$4 \quad$ V407 Cyg as a gamma-ray transient}

Fermi LAT detection of a New Galactic Plane Gamma-ray Transient in the Cygnus Region: Fermi J2102+4542, and its Possible Association with V407 Cyg was reported in ATel 2487; C. C. Cheung (NRC/NRL), D. Donato (NASA GSFC), E. Wallace (U. Washington), R. Corbet (NASA GSFC), G. Dubus (U. Grenoble), K. Sokolovsky (MPIfR), H. Takahashi (Hiroshima U.) and by Abdo et al. (2010) on behalf of the Fermi Large Area Telescope Collaboration. The Large Area Telescope (LAT), on board the Fermi Gamma-ray Space Telescope, detected a transient gamma-ray source in the Galactic Plane: Fermi J2102+4542. Preliminary analysis of the Fermi-LAT data indicates that on the 13th and 14th of March 2010, the source was detected with a more than $100 \mathrm{MeV}$ flux of $(1.0 \pm 0.3) \times 10^{-6} \mathrm{ph}$ 

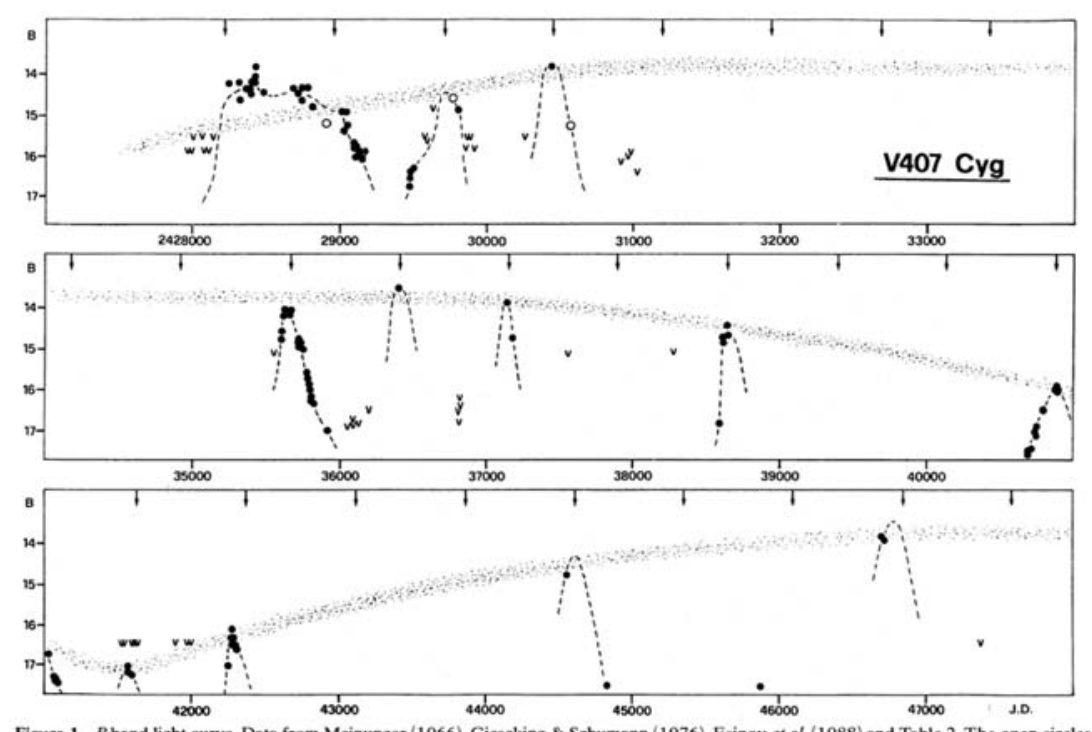

Figure 1. B band light curve, Data from Meinunger (1966), Gieseking \& Schumann (1976), Esipov et al. (1988) and Table 2. The open circles indicate lower weight points in Meinunger's data. The dotted line is a hand-drawn interpolation of the maxima showing the effect of the dust

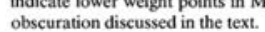

Fig. 1: The historical light curve of V407 Cyg (Munari et al., 1990)

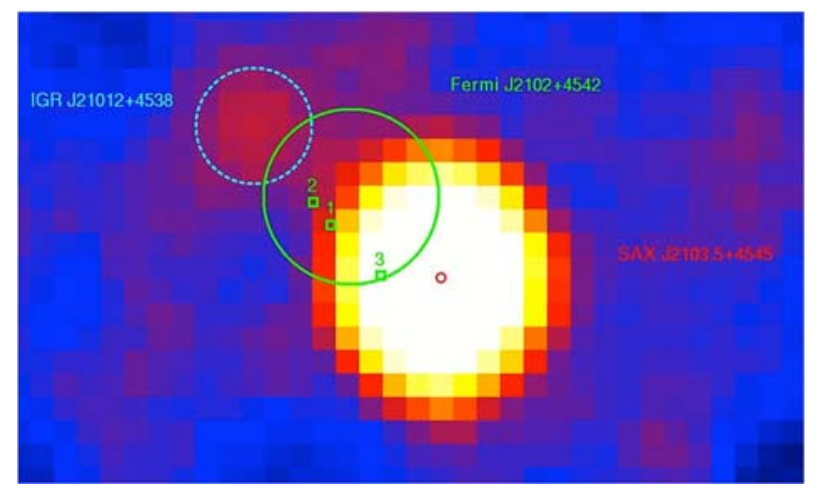

Fig. 2: INTEGRAL IBIS observation of the V407 Cyg position (Bassani, 2010) shows the new transient to be located between two persistent hard X-ray sources, with no confirmation of the transient detection

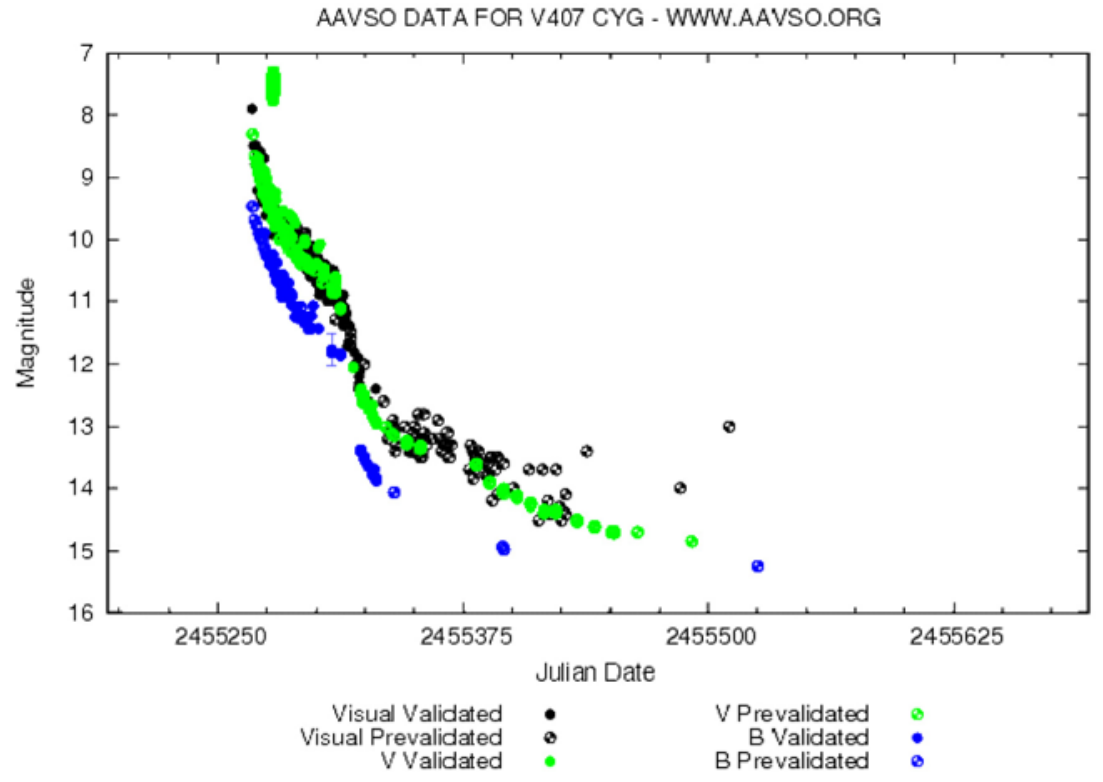

Fig. 3: V407 Cyg optical CCD observations prior to and after the 2010 optical flare (courtesy of AAVSO http://www.aavso.org) 


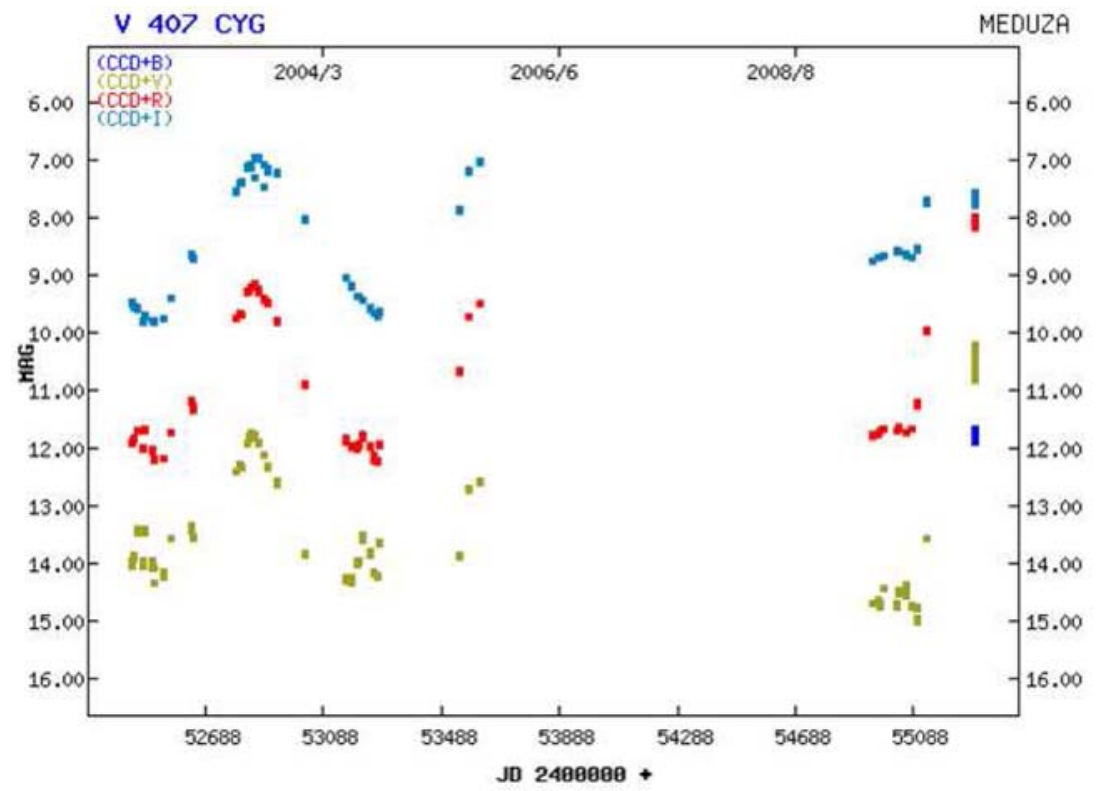

Fig. 4: V407 Cyg optical CCD observations prior to the 2010 optical flare (courtesy of Meduza, Lubos Brat, Czech Republic)

$\mathrm{cm}^{-2} \cdot \mathrm{s}^{-1}$ and $(1.4 \pm 0.4) \times 10^{-6} \mathrm{ph} \mathrm{cm}^{-2} \cdot \mathrm{s}^{-1}$, respectively (statistical only) — the corresponding significances on these days are 8 sigma and 6 sigma. A systematic uncertainty of $30 \%$ should be added to this number. There is no previously reported gamma-ray source at this location.

Within the $95 \%$ confidence error circle radius of $0.12 \mathrm{deg}$ (statistical only) is the symbiotic star V407 Cyg, with a reported optical outburst beginning approximately 2 days earlier (CBET 2199) than the onset of gamma-ray activity detected by LAT. Swift/XRT observations triggered on the optical outburst of V407 Cyg and performed on 2010 March 13 th and 15 th resulted in 2.4-2.6 sigma $(0.3-10 \mathrm{keV})$ detections of an X-ray source coincident with the position of the star in each of the two $960 \mathrm{sec}$ exposures.

The gamma-rays could have been caused by shock driven acceleration of the ejected material, and its capture by strong magnetic fields within the system. This is the first hard gamma-ray emission detected from this category of objects.

\section{The case of FY Aql}

Other Mira/Symbiotics stars with possible gammaray eruption/association have also been known, but without firm confirmation. One of these candidates is the variable star FY Aql located inside the error box of GRB19790331.

An optical study in the field of FY Aql/ GRB19790331 was published by Hudec in 1989. The investigated Mira/Symbiotics star FY Aql is inside a small error box of the gamma-ray burst (GRB)
GRB19790331. Results were presented from an optical investigation of the error box of the gamma-ray burst source (GRBS) 1979 0331. Consideration was given to the light changes and the optical behavior of the variable FY Aql, located inside the box. These results confirm that FY Aql is indeed a Mira variable with short period flares.

This Mira/Symbiotics system had a temporary reflecting nebula, indicating possible eruption in the past. The object position is in a crowded part of the Milky Way in Aquila. During a standard catalog search of the box, Laros et al. (1985) found that the Mira variable star FY Aql is inside the region. Subsequent studies have found that the Mira star has short-duration flares and a surrounding reflection nebula, while the nebula has subsequently disappeared. Details concerning FY Aql are given in Hartmann and Pogge (1987), Hudec (1989), Schaefer et al. (1987), Hudec (1987), Schaefer (1990), Schaefer (1991), and Irwin and Zytkow (1994).

\section{Conclusions}

The symbiotics Mira variable star V407 Cygni is very probably coincident with a gamma-ray transient detected by Fermi. There are also other objects in this category that may be sources of high-energy gammaray emmission, such as FY Aql. Obviously the category of Mira Symbiotic variables with reflecting (temporary) nebulas are worth further study to establish the physics behind the posible gamma-ray activity. The spectroscopic properties during the optical flare indicate the possibility to detect such events 
by RP/BP photometers/ultra-low resolution spectrographs on ESA Gaia satellite. These findings also confirm the importance of long-term optical monitoring by CCD telescopes and cameras and also historical long-term optical coverage provided by astronomical plate collections.

\section{Acknowledgement}

I ackowledge grants 205/08/1207 and 102/09/0997 provided by the Grant Agency of the Czech Republic and by MSMT KONTAKT Project ME09027. The CCD observations of V407 prior to the 2010 flare were provided by Meduza collaboration/Lubos Brat, Czech Republic.

\section{References}

[1] Abdo, A. A., et al.: Science, Vol. 329, Issue 5993, pp. 817-821, 2010.

[2] Bassani, A., et al.: ATEL2498, 2010.

[3] Hartmann, D., Pogge, R. W.: ApJ, 318, 363, 1987.

[4] Hoffmeister, C.: VVS 1, 1949, 295.

[5] Nishiyama, Kabashima: CBET, 2010, 2199.

[6] Irwin, M., Zytkow, A.: ApJ, 433, L81, 1994.

[7] Laros, J. G., et al.: ApJ, 290, 728, 1985.

[8] Munari, U., et al.: CBET, 2010, 2204.
[9] Munari, U., et al.: Monthly Notices of the Royal Astronomical Society: Letters, Vol. 410, Issue 1, pp. L52-L56, 2011.

[10] Cheung, C. C., et al.: ATel, 2010, 2487.

[11] Hudec, R.: IBVS, $\mathbf{3}$ 121, 1, 1987.

[12] Hudec, R., Bull: Astronomical Institutes of Czechoslovakia, Vol. 40, No. 4, Aug. 1989, p. 261-266.

[13] Meinunger, L.: MVS Sonneberg, 3, 111, 1966.

[14] Munari, U., et al.: MNRAS, 1990, 242, 653.

[15] Schaefer, B. E.: ApJ, 364, 590, 1990.

[16] Schaefer, B. E.: ApJ, 366, L39, 1991.

[17] Shugarov, S. Y., Tatarnikova, A. A., Kolotilov, E. A., Shenavrin, V. I., Yudin, B. F.: V407 CYG as a Member of a New Subclass of Symbiotic Stars. Baltic Astronomy, Vol. 16, p. 23-25, 1997.

[18] Shore, N. S., et al.: Astronomy \& Astrophysics, Vol. 527, id.A98, 2011.

René Hudec

E-mail: rhudec@asu.cas.cz

Astronomical Institute

Academy of Sciences of the Czech Republic

CZ-251 65 Ondřejov, Czech Republic

Czech Technical University in Prague

Faculty of Electrical Engineering

Technická 2, CZ-166 27 Prague, Czech Republic 